\title{
Direct Observation of Electron Distributions inside Millisecond Duration Electron Holes
}

\author{
F. S. Mozer, ${ }^{1, *}$ O. V. Agapitov, ${ }^{1}$ B. Giles, ${ }^{2}$ and I. Vasko ${ }^{1}$ \\ ${ }^{1}$ Space Sciences Laboratory, University of California, Berkeley, California 94720, USA \\ ${ }^{2}$ NASA Goddard Space Flight Center, Greenbelt, Maryland 20771, USA
}

(Received 4 June 2018; published 27 September 2018)

\begin{abstract}
Despite the importance of millisecond duration spatial structures [chorus wave nonlinearity or time domain structures (TDS)] to plasma dynamics, there have been no direct observations of the generation and interaction of these waves and TDS with electrons at the millisecond timescale required for their understanding. Through superposition of 0.195 ms Magnetospheric Multiscale Satellite electron measurements inside 37 superposed, millisecond duration electron holes, the first observations of electron spectra and pitch angle distributions on a submillisecond timescale have been obtained. They show that $\mathrm{keV}$ electrons inside the superposed electron hole are accelerated by several hundred volts and that the spectrum of electrons inside the electron hole contain several maxima and minima that are explained by a model of electron energy changes on entering the holes. We report the first observation of trapped electrons inside the TDS, in accordance with the theoretical requirement that such electrons must be present in order to form the phase space holes. Mechanisms of electron acceleration by electron holes (through perpendicular energy gain as the TDS moves into a converging magnetic field) and scattering (due to the perpendicular electric field) are discussed.
\end{abstract}

DOI: 10.1103/PhysRevLett.121.135102

Introduction.-On September 27, 2016 at 01:19:37 UT, the four Magnetospheric Multiscale Satellite (MMS) spacecraft were separated by about $6 \mathrm{~km}$ in the dusk magnetosphere at Geocentric Solar Ecliptic Coordinates $(-2.5,10.0,0.6)$ Earth radii when an injection of enhanced flowing plasma accompanied by a strong field-aligned current and low frequency electrostatic turbulence occurred. The magnetic field was $64 \mathrm{nT}$, the density was $0.15 \mathrm{~cm}^{-3}$, the electron temperature was $1000 \mathrm{eV}$, and the field-aligned current was about $0.05 \mu \mathrm{A} / \mathrm{cm}^{2}$. The turbulence consisted of bipolar parallel electric field structures, $\sim 20$ of which in $150 \mathrm{~ms}$ are the subject of this Letter. These structures moved at about $10000 \mathrm{~km} / \mathrm{s}$ (a fraction of the electron thermal speed), and they contained diverging parallel electric fields and positive peak electrostatic potentials with little or no net parallel potential. These properties allow the structures to be identified as electron phase space holes. Their existence is due to phase space density deficits of electrons trapped within the bipolar electric field [1]. They were likely formed in a nonlinear stage of an electron streaming beam instability $[2,3]$ because the electron phase space density had a plateau in the corresponding energy range.

Wave-particle interactions, such as those occurring with these millisecond time domain structures, are responsible for electron heating and acceleration in magnetospheres, solar winds, and astrophysics. For example, the cyclotron resonance of $\sim 1 \mathrm{kHz}$ chorus waves is related to pulsating auroras [4], microbursts [5], and electron acceleration to relativistic energies in Earth's radiation belts [6]. Time domain structures (electron holes, double layers, electron acoustic solitons, ion acoustic solitons, the Landau resonance of nonlinear chorus waves, etc.) appear in enormous quantities in Earth's radiation belts [7], in the solar wind [8], in the bow shock [9], at reconnection sites [10], and at other planets [11]. They are involved in electron thermalization at shocks [12], electron acceleration in pulsating auroras [13], electron heating and acceleration in the radiation belts [14-18], and electron acceleration to relativistic energies $[19,20]$. They have also been generated in laboratory plasmas [21].

Theoretical mechanisms for generation of chorus waves and time domain structures (TDS) and their electron scattering, heating, and acceleration are well documented in the literature [22-46].

Despite this extensive work and the importance of these millisecond structures to plasma dynamics, there have been no direct observations of the generation and interaction of these waves and TDS with plasma electrons at the required millisecond timescale. Because the MMS mission measures electrons every $0.195 \mathrm{~ms}$, its data can yield information on electron distributions in the millisecond durations of TDS structures such as those on September 27, 2016. We have examined the electrons inside these streams of electron holes to find that both expected and unexpected features of the electron distributions occur on this short timescale.

Data processing and analysis.-MMS measures electrons at a single energy, 16 polar angles and 8 azimuthal angles every $0.195 \mathrm{~ms}$ [47]. In successive $0.195 \mathrm{~ms}$ intervals, the measured energy is stepped such that a 

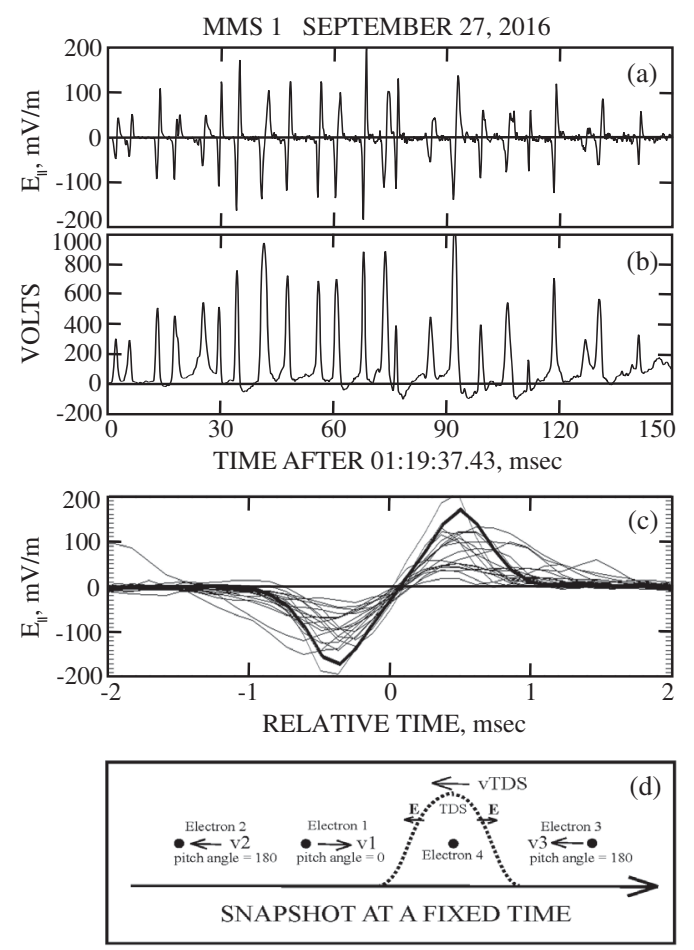

FIG. 1. (a) A stream of electron holes observed on MMS1 during a period of about $150 \mathrm{~ms}$. The integrated potential is presented in (b), while the superposition of the electron holes is illustrated in (c). A snapshot at a fixed time of the superposed electron hole potential and specific electrons is described in (d).

complete 32 point energy distribution is measured every $7.5 \mathrm{~ms}$. However, $7.5 \mathrm{~ms}$ is long compared to the less-than1-ms duration of a single cycle of a chorus wave or the duration of a typical TDS. Thus, to observe a full energy spectrum in less than $1 \mathrm{~ms}$, such $0.195 \mathrm{~ms}$ electron measurements from successive wave periods or successive TDS must be combined in a superposed epoch analysis. This superposition technique assumes that the electrons entering the different TDS are similar and interact with the TDS in similar manners even though the TDS have different amplitudes (see Fig. 1). That the entering electrons have not been affected by their interactions with previous TDS is shown in a later figure.

The data for doing the superposition are described in Fig. 1(a), which illustrates $150 \mathrm{~ms}$ of the parallel (to the background magnetic field) electric field measured on MMS1. The data rate for these measurements was 8192 samples/s. The same TDS structures were observed on the three other MMS spacecraft, which allowed measurement of their $\sim 10000 \mathrm{~km} / \mathrm{s}$ speed antiparallel to the local quasistatic magnetic field direction. Their amplitudes varied from about 50 to $200 \mathrm{mV} / \mathrm{m}$, and their integrated peak potentials illustrated in Fig. 1(b) were 200 to 1000 V, although their net potentials were small. These electron holes have parallel electric fields pointing in the negative magnetic field direction at their fronts and along the magnetic field direction at their trailing edges. Figure 1(c) presents the superposition of these TDS, which is required to obtain measurements at all energies, as described above.

There are about 20 electron holes illustrated in Fig. 1, about ten of which had peak potentials greater than $500 \mathrm{~V}$. The superposition of data from the four spacecraft included 37 electron holes with peak potentials greater than $500 \mathrm{~V}$. Each millisecond electron hole covered electron data at four energies. Thus, 150 measurements were made inside the electron holes at 32 energies, giving an average of five measurements at each energy. Each such energy measurement included 128 different pitch angles. Thus, there are sufficient measurements at all energies and pitch angles inside the superposed electron hole to produce statistically significant results.

Electrons with different pitch angles and energies interact with the TDS in different ways, as illustrated in Fig. 1(d), which is a snapshot at a fixed time with the TDS potential moving to the left, antiparallel to the magnetic field. Also illustrated are four electrons that interact with the TDS in different ways. All electrons of type 1 having small pitch angles move opposite to the TDS, so they enter the TDS and are accelerated by its electric fields. Electrons 2 , at large pitch angles, can only interact with the TDS if their speed is less than that of the TDS. Electrons of type 3 interact with the TDS only if their speeds are greater than that of the TDS. Electrons of type 4 are trapped inside the TDS parallel electric fields at its boundaries. The results of these interactions of the various types of electrons with the TDS are illustrated in the following plots.

Figure 2 presents energy spectra produced from this data set (electrons with energies below $100 \mathrm{eV}$ are not included because of spurious signatures at low energies from the photoelectric effect, scattering in and near the detector aperture, etc.) Each spectrum is composed from 2049 individual measurements. Figure 2(a) shows spectra of $150^{\circ}-180^{\circ}$ pitch angle electrons outside and inside the TDS, where inside is defined as being within $\pm 0.25 \mathrm{~ms}$ of the center of the superposed TDS (which is the width in time between the peaks of the TDS of Fig. 1) and outside means the average of the data during the $1 \mathrm{~ms}$ before and after the TDS. Figure 2 (a) shows that $150^{\circ}-180^{\circ}$ electrons exhibit a plateau outside of the TDS in the energy range of $100 \mathrm{eV}-2 \mathrm{keV}$. This indicates that the TDS are likely produced by a beam instability, and the plateau energy range is in agreement with the estimated TDS velocities. Inside the TDS, electrons in the same energy range exhibit a deficit of the phase space density. This deficit is strictly required for electron holes to exist according to theory [1], and Fig. 2(a) presents the first experimental evidence in the literature for this prediction of the theory.

Figure 2(b) presents the same data for $0^{\circ}-30^{\circ}$ pitch angle electrons. For both these large and small pitch angle ranges, electrons with energies above a few $\mathrm{keV}$ were accelerated as they moved inside the TDS structure. (It is noted that 


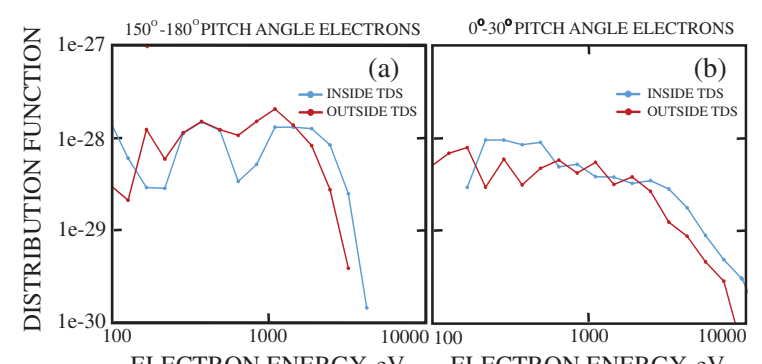

ELECTRON ENERGY, eV ELECTRON ENERGY, eV

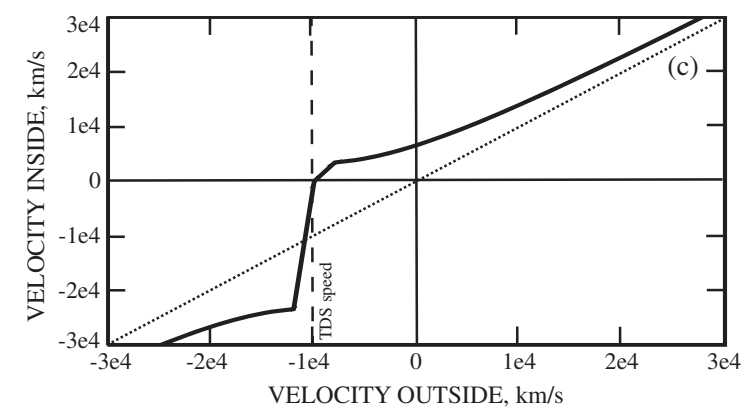

FIG. 2. Spectra of large (a) and small (b) pitch angle electrons outside and inside the superposed electron hole. Panel (c) presents the electron parallel velocity inside the TDS as a function of its velocity outside the TDS for a model in which the TDS speed is $-10000 \mathrm{~km} / \mathrm{s}$ and its peak potential is $500 \mathrm{~V}$.

they were also decelerated by a similar amount on exiting the TDS, so the outside spectra on either side of the TDS were essentially identical).

The $0^{\circ}-30^{\circ}$ electrons traveled in the direction opposite to that of the TDS, so they had a head-on collision. To compute the electron energy gain in a model of this collision, the interaction is viewed in the frame of the TDS, in which the electrons saw a static accelerating potential of $500 \mathrm{~V}$. Thus, they gained $500 \mathrm{eV}$ of energy in this frame, which, when transformed back to the spacecraft frame, is an average energy gain of $310 \mathrm{eV}$ for 1000-10 $000 \mathrm{eV}$ electrons. This estimate compares with the observed $400 \mathrm{eV}$ gain. Given the wide spread in the individual TDS peak potentials displayed in Fig. 1(b), this is a satisfactory agreement.

The $150^{\circ}-180^{\circ}$ electrons have a different interaction because they traveled in the same direction as the TDS. If their speed was greater than that of the TDS, they overtook the TDS and were accelerated by its attractive potential inside the TDS. Electrons with energies greater than $200 \mathrm{eV}$ had such speeds and their energy gain inside the TDS computed as described for the $20^{\circ}$ electrons averaged $540 \mathrm{eV}$. This compares with the observed average energization of $480 \mathrm{eV}$. It is noted that this discussion concerns comparisons of spectra inside and outside the TDS and that before and after the TDS, the electron spectra were unchanged.

A model calculation of the electron parallel velocity inside a TDS as a function of its parallel velocity outside the TDS is given in Fig. 2(c) for the case of the TDS moving at
$-10000 \mathrm{~km} / \mathrm{s}$ with a $500 \mathrm{~V}$ peak potential. As discussed above and observed in the data, the model of Fig. 2(c) shows acceleration of $\mathrm{keV}$ electrons traveling in both directions. It also shows a special behavior for an outside electron moving at a speed near that of the TDS [the dashed vertical line in Fig. 2(c)]. An outside electron with a speed slightly more negative than the TDS speed and overtaking the TDS has a speed inside the TDS that is more than a factor of 2 larger in magnitude. Thus, there should be fewer electrons at $150^{\circ}-180^{\circ}$ with energies somewhat above a few hundred $\mathrm{eV}$ (the energy associated with the TDS speed) than elsewhere in the electron spectrum. This explains the observed minimum between about 400 and $1000 \mathrm{eV}$ in Fig. 2(a).

The Fig. 2(b) 200-500 eV maximum in the electron distribution inside the TDS relative to that outside and the minimum at somewhat smaller energies in Fig. 2(a) are also explained in the model of Fig. 2(c) as follows. In this model, an outside electron with a negative parallel velocity between zero and the TDS speed becomes a positive velocity electron inside the TDS. Thus, it is removed from the $150^{\circ}-180^{\circ}$ spectrum and added to the $0^{\circ}-30^{\circ}$ spectrum. In this way, the spectra and their variations in Fig. 2 are well understood in terms of a model of the process that they undergo.

The data set consisting of 59263 counter outputs provides further insights into electron interactions with the TDS in the Fig. 3 plot of the "count rate" around the TDS as a function of the electron parallel velocity. This count rate is obtained from a plot of the phase space density in the parallel-perpendicular velocity plane by integrating (actually summing) over the perpendicular velocity. This makes the count rate proportional to the phase space density times the energy times the square of the sine of the pitch angle. The egg-shaped structure in Fig. 3 centered at the TDS speed of about $-10000 \mathrm{~km} / \mathrm{s}$ and bounded in height by the $500 \mathrm{~V}$ TDS potential and in width by the \pm 1 ms duration of the TDS defines the region within which all electrons are trapped inside the TDS parallel electric field. This is because all passing electrons are accelerated by the TDS, as seen in the figure, to have parallel velocities outside this egg-shaped region. It is noted that the parallel

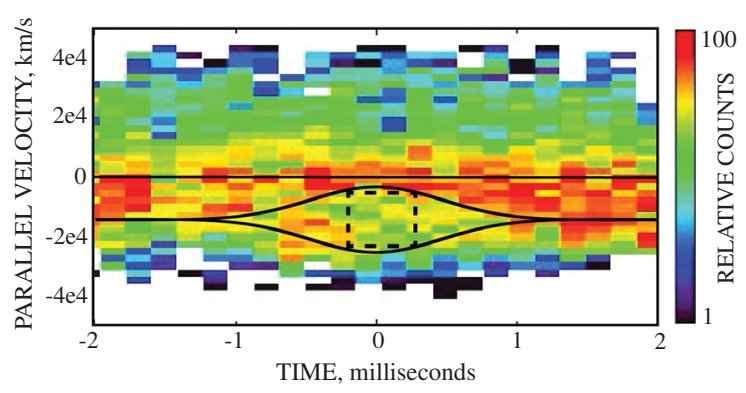

FIG. 3. The 56-5471 eV electron velocity distribution in space around the superposed TDS. 


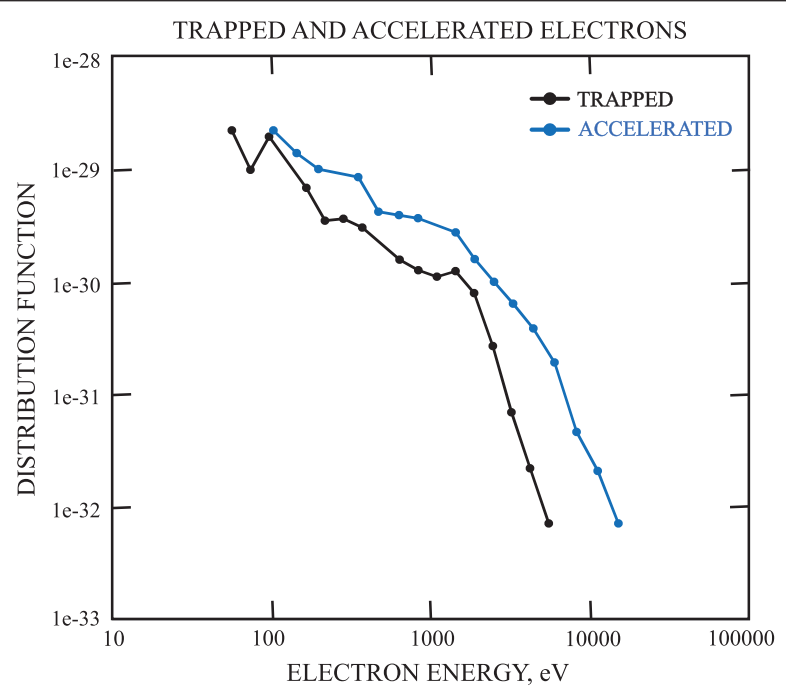

FIG. 4. Distribution functions of electrons trapped in the electron hole.

electron distributions at the beginning and end of the $4 \mathrm{~ms}$ interval in Fig. 3 are similar, which suggests that the electrons in the TDS of interest were not influenced by interactions with earlier TDS.

The phase space density of the trapped electrons inside the small dashed rectangle of Fig. 3 is plotted in Fig. 4. There is no upper limit on the trapped electron energy because very energetic electrons with near $90^{\circ}$ pitch angles can satisfy the parallel velocity requirements to become trapped. If the electrons trapped in the observed electron holes were to remain trapped as the structure moved from the equator to $30^{\circ}$ latitude, the flux of several $\mathrm{keV}$ electrons conserving the first adiabatic invariant, would increase by orders of magnitude as the magnitude of the magnetic field increased by a factor of about 3 . Such accelerated electrons are also plotted in Fig. 4. This is a powerful acceleration mechanism.

Discussion.-Through data analyses and modeling of electron distributions inside and outside of a superposed electron hole, the properties of electrons passing through or trapped by the electron hole have been measured on a submillisecond timescale. The distribution function of trapped electrons has been measured for the first time, and spectral structures in both the large and small pitch angle data have been understood in terms of a model of electron acceleration by a $10000 \mathrm{~km} / \mathrm{s}, 500 \mathrm{~V}$ peak potential electron hole. Because these results show the validity of the approach of superposing data from many millisecond structures to obtain full energy coverage and adequate counting statistics, much additional work remains to be done. For example, because electrons may be scattered or accelerated by the perpendicular electric fields in the TDS, it would be interesting to compare electron properties for TDS having important perpendicular electric fields with those having small perpendicular fields. Work such as this is planned for the future.
This Letter has not discussed the growth of TDS or the effect of the observed electrons on the TDS structure and lifetime. While these are crucial questions that must be analyzed in order to understand these wave-particle interactions, such questions are beyond the scope of this work.

The authors thank the entire Magnetospheric Multiscale Satellites (MMS) team for providing such excellent data. This work was supported by Johns Hopkins University/ Applied Physics Lab Contract No. 922613 (Radiation Belt Storm Probes-Electric Fields and Waves) and MMS Guest Investigator Grant No. 16-MMSGI16-2-0021.

* Corresponding author. forrest.mozer@gmail.com

[1] H. Schamel, Electron holes, ion holes and double layers: Electrostatic phase space structures in theory and experiment, Phys. Rep. 140, 161 (1986).

[2] R. L. Morse and C. W. Nielson, One-, Two-, and ThreeDimensional Numerical Simulation of Two-Beam Plasmas, Phys. Rev. Lett. 23, 1087 (1969).

[3] Y. Omura, H. Matsumoto, T. Miyake, and H. Kojima, Electron beam instabilities as generation mechanism of electrostatic solitary waves in the magnetotail, J. Geophys. Res. 101, 2685 (1996).

[4] Y. Nishimura, J. Bortnik, W. Li et al., Identifying the driver of pulsating aurora, Science 330, 81 (2010).

[5] F. S. Mozer, O. V. Agapitov, J. B. Blake, and I. Y. Vasko, Simultaneous observations of lower band chorus emissions at the equator and microburst precipitating electrons in the ionosphere, Geophys. Res. Lett. 45, 511 (2018).

[6] R. S. Selesnick and J. B. Blake, On the source location of radiation belt relativistic electrons, J. Geophys. Res. 105, 2607 (2000).

[7] F. S. Mozer, S. D. Bale, J. W. Bonnell, C. C. Chaston, I. Roth, and J. Wygant, Megavolt Parallel Potentials Arising from Double-Layer Streams in the Earth's Outer Radiation Belt, Phys. Rev. Lett. 111, 235002 (2013).

[8] S. D. Bale, D. Burgess, P. J. Kellogg et al., Phase coupling in Langmuir wave packets: Possible evidence of three-wave interactions in the upstream solar wind, Geophys Lett. 23, 109 (1996).

[9] S. D. Bale, P. J. Kellogg, D. E. Larsen, R. P. Lin, K. Goetz, and R. P. Lepping, Bipolar electrostatic structures in the shock transition region: Evidence of electron phase space holes, Geophys. Res. Lett. 25, 2929 (1998).

[10] F. S. Mozer, O. A. Agapitov, A. Artemyev, J. L. Burch, R. E. Ergun, B. L. Giles, D. Mourenas, R. B. Torbert, T. D. Phan, and I. Vasko, Magnetospheric Multiscale Satellite Observations of Parallel Electron Acceleration in Magnetic Field Reconnection by Fermi Reflection from Time Domain Structures, Phys. Rev. Lett. 116, 145101 (2016).

[11] J. S. Pickett, W. S. Kurth, D. A. Gurnett, R. L. Huff, J. B. Faden, F. F. Averkamp, D. Pisa, and G. H. Jones, Electrostatic solitary waves observed at Saturn by Cassini inside 10 Rs and near Enceladus, J. Geophys. Res. 120, 6569 (2015).

[12] I. Y. Vasko, F. S. Mozer, V. V. Krasnoselskikh, A. V. Artemyev, O. V. Agapitov, S. D. Bale, T. Phan, R. Ergun, 
B. Giles, D. Gershman, P.-A. Lindqvist, C. T. Russell, R. Strangeway, and R. B. Torbert, Solitary waves across supercritical quasi-perpendicular shocks, Geophys. Res. Lett. 45, 5809 (2018).

[13] F. S. Mozer, O. V. Agapitov, A. Hull, S. Lejosne, and I. Y. Vasko, Pulsating auroras produced by interactions of electrons and time domain structures, J. Geophys. Res. Space Phys. 122, 8604 (2017).

[14] A. Artemyev, O. V. Agapitov, F. S. Mozer, and V. Krasnoselskikh, Thermal electron acceleration by localized bursts of electric field in the radiation belts, Geophys. Res. Lett. 41, 5734 (2014).

[15] I. Y. Vasko, O. V. Agapitov, F. S. Mozer, and A. V. Artemyev, Thermal electron acceleration by electric field spikes in the outer radiation belt: Generation of field-aligned pitch angle distributions, J. Geophys. Res. 120, 8616 (2015).

[16] I. V. Vasko, O. V. Agapitov, F. S. Mozer et al., Electron holes in inhomogeneous magnetic field: Electron heating and electron hole evolution, Phys. Plasmas 23, 052306 (2016).

[17] I. Y. Vasko, O. V. Agapitov, F. S. Mozer, A. V. Artemyev, V. V. Krasnoselskikh, and J. W. Bonnell, Diffusive scattering of electrons by electron holes around injection fronts, J. Geophys. Res. 122, 3163 (2017).

[18] I. Y. Vasko, O. V. Agapitov, F. S. Mozer, A. V. Artemyev, J. F. Drake, and I. V. Kuzichev Electron holes in the outer radiation belt: Characteristics and their role in electron energization, J. Geophys. Res. 122, 120 (2017).

[19] F. S. Mozer, O. V. Agapitov, V. Krasnoselskikh, S. Lejosne, G. D. Reeves, and I. Roth, Direct Observation of RadiationBelt Electron Acceleration from Electron-Volt Energies to Megavolts by Nonlinear Whistlers, Phys. Rev. Lett. 113, 035001 (2014).

[20] F. S. Mozer, A. Artemyev, O. V. Agapitov, D. Mourenas, and I. Vasko, Near-relativistic electron acceleration by Landau trapping in time domain structures, Geophys. Res. Lett. 43, 508 (2016).

[21] W. Fox, M. Porkolab, J. Egedal, N. Katz, and A. Le, Laboratory Observation of Electron Phase-Space Holes during Magnetic Reconnection, Phys. Rev. Lett. 101, 255003 (2008).

[22] O. V. Agapitov, A. Artemyev, D. Mourenas, V. Krasnoselskikh, J. Bonnell, O. Le Contel, C. M. Cully, and V. Angelopoulos, The quasi-electrostatic mode of chorus waves and electron nonlinear acceleration, J. Geophys. Res. 119, 1606 (2014).

[23] O. V. Agapitov, V. Krasnoselskikh, F. S. Mozer, A. V. Artemyev, and A.S. Volokitin, Generation of nonlinear electric field bursts in the outer radiation belt through the parametric decay of whistler waves, Geophys. Res. Lett. 42, 3715 (2015).

[24] O. Agapitov, J. F. Drake, I. Vasko, and F. S. Mozer, Nonlinear electrostatic steepening of whistler waves: The guiding factors and dynamics in inhomogeneous systems, Geophys. Res. Lett. 45, 2168 (2018).

[25] M. Berthomier, R. Pottelette, and M. Malingre, Solitary waves and weak double layers in a two-electron temperature auroral plasma, J. Geophys. Res. 103, 4261 (1998).
[26] J. Drake, O. Agapitov, and F. Mozer, The development of a bursty precipitation front with intense localized parallel electric fields driven by whistler waves, Geophys. Res. Lett. 42, 2563 (2015).

[27] M. V. Goldman, M. M. Oppenheim, and D. L. Newman, Nonlinear two-stream instabilities as an explanation for auroral bipolar wave structures, Geophys. Res. Lett. 26, 1821 (1999).

[28] R. F. Hubbard and G. Joyce, Simulation of aurora1 double layers,, J. Geophys. Res. 84, 4297 (1979).

[29] I. H. Hutchinson, Electron holes in phase space: What they are and why they matter, Phys. Plasmas 24, 055601 (2017).

[30] G. Joyce and R.F. Hubbard, Numerical simulation of plasma double layers, J. Plasma Phys. 20, 391 (1978).

[31] V. I. Karpman and R. N. Kaufman, Self-focusing of whistlers waves, Zh. Eksp. Teor. Fiz. 83 (1982) [Physics of Fluids B: Plasma Physics 4, 3087 (1992)].

[32] P. J. Kellogg, C. A. Cattell, K. Goetz, S. J. Monson, and L. B. Wilson, III., Electron trapping and charge transport by large amplitude whistlers, Geophys. Res. Lett. 37, L2016 (2010).

[33] I. V. Kuzichev, I. Y. Vasko, O. V. Agapitov et al., Evolution of electron phase space holes in inhomogeneous magnetic fields, Geophys. Res. Lett. 44, 2105 (2017).

[34] C. Lashmore-Davies and T. Martin, Electrostatic instabilities driven by an electric current perpendicular to a magnetic field, Nucl. Fusion 13, 193 (1973).

[35] T. Miyake, Y. Omura, H. Matsumoto, and H. Kojima, Twodimensional computer simulations of electrostatic solitary waves observed by Geotail spacecraft, J. Geophys. Res. 103, 11841 (1998).

[36] F. S. Mozer, O. V. Agapitov, A. Artemyev, J. F. Drake, V. Krasnoselskikh, S. Lejosne, and I. Vasko, Time domain structures: What and where they are, what they do, and how they are made, Geophys. Res. Lett. 42, 3627 (2015).

[37] D. L. Newman, M. V. Goldman, R. E. Ergun, and A. Mangeney, Formation of Double Layers and Electron Holes in a Current Driven Space Plasma, Phys. Rev. Lett. 87, 255001 (2001).

[38] Y. Omura, Y. Katoh, and D. Summers, Theory and simulation of the generation of whistler-mode chorus, J. Geophys. Res. 113, A04223 (2008).

[39] Y. Omura, D. Nunn, and D. Summers, in Generation Processes of Whistler Mode Chorus Missions: Current Status of Nonlinear Wave Growth Theory, Geophysical Monograph Series, edited by D. Summers, I. U. Mann, D. N. Baker, and M. Schulz (American Geophysical Union, Washington, DC, 2013).

[40] M. M. Oppenheim, G. Vetoulis, D. L. Newman, and M. V. Goldman, Evolution of electron phase-space, holes in 3D, Geophys. Res. Lett. 28, 1891 (2001).

[41] V.D. Shapiro and S. K. Ride, Nonlinear effects involving whistler wave propagation in the magnetosphere, J. Geophys. Res. 99, 17237 (1994).

[42] N. Singh, Dynamically evolving double layers and density depletions, J. Geophys. Res. 108 (2003). 
[43] I. V. Vasko, I. V. Kuzichev, O. V. Agapitov et al. Evolution of electron phase space holes in inhomogeneous plasmas, Phys. Plasmas 24, 062311 (2017).

[44] I. V. Vasko, O. V. Agapitov, F. S. Mozer, J. W. Bonnell, A. V. Artemyev, V. V. Krasnoselskikh, G. Reeves, and G. Hospodarsky, Electron-acoustic solitons and double layers in the inner magnetosphere, Geophys. Res. Lett. 44, 4575 (2017).
[45] I. Y. Vasko, O. V. Agapitov, F. S. Mozer, J. W. Bonnell, A. V. Artemyev, V. V. Krasnoselskikh, and Y. Tong, Electrostatic Steepening of Whistler Waves, Phys. Rev. Lett. 120, 195101 (2018).

[46] T. Yamamoto and J. R. Kan, Double layer formation due to current injection, Planet. Space Sci. 33, 853 (1985).

[47] C. Pollock et al., Fast plasma investigation for magnetospheric multiscale, Space Sci. Rev. 199, 331 (2016). 